\title{
Synthesis and Effect of Lattice Strain on the Debye-Waller Factors of Zinc Nanoparticles
}

\author{
Endla Purushotham \\ Department of Physics, Humanities and Science, S R Engineering College (Autonomous), Warangal, India
}

Email address:

psm45456@gmail.com

\section{To cite this article:}

Endla Purushotham. Synthesis and Effect of Lattice Strain on the Debye-Waller Factors of Zinc Nanoparticles. Modern Chemistry. Vol. 7, No. 1, 2019, pp. 5-9. doi: 10.11648/j.mc.20190701.12

Received: December 18, 2018; Accepted: January 20, 2019; Published: January 31, 2019

\begin{abstract}
Zn nanopowder was prepared by high-energy ball milling has been investigated. Zn powders were ball milled in an argon inert atmosphere. The milled powders were characterized by X-ray diffraction and scanning electron microscopy measurements. Lattice strains in Zn powders produced by milling have been analyzed by X-ray powder diffraction. The lattice strain $(\varepsilon)$ and Debye-Waller factor (B) are determined from the half-widths and integrated intensities of the Bragg reflections. Debye-Waller factor is found to increase with the lattice strain. From the correlation between the strain and effective DebyeWaller factors have been estimated for $\mathrm{Zn}$. The variation of energy of vacancy formation as a function of lattice strain has been studied.
\end{abstract}

Keywords: Ball Milling, X-Ray Diffraction, Particle Size, Lattice Strain, Debye-Waller Factor, Vacancy Formation Energy

\section{Introduction}

Synthesis of nanomaterials are important for further miniaturization of electric devices, solar cells to enhance efficiency of heat engines, as chemical catalysts $[1,4]$. Physical and chemical properties of these materials are highly size dependent. Therefore, it is important to develop novel techniques for the synthesis of nanomaterials. The Debye-Waller factor is an important lattice dynamical property. There is considerable X-ray work on the DebyeWaller factors of $\mathrm{Zn} \mathrm{[5-6].} \mathrm{But} \mathrm{it} \mathrm{is} \mathrm{interesting} \mathrm{to} \mathrm{study} \mathrm{the}$ effect of particle size and lattice strains on the Debye-Waller factors of these metals. Inagaki et al $[7,8]$ showed that in several non-metallic powders, the strains produced during grinding have a significant effect on the Debye-Waller factors measured from X-ray diffraction intensities. Sirdeshmukh et al [9] observed the effect of lattice strains on the DebyeWaller factors in semiconductor powder materials. Gopi Krishna and Sirdeshmukh [10] studied the effect of lattice strains on the Debye-Waller factor of ytterbium metal. Gopi Krishna et al [11] studied the effect of lattice strains on the Debye-Waller factor of $\mathrm{Mg}, \mathrm{Zn}$ and $\mathrm{Cd}$ for slow grinding in an agate mortar. In the present investigation the results of a systematic study of the effect of particle size and lattice strains on the Debye-Waller factors of hexagonal $\mathrm{Zn}$ metal powder is reported using ball mill. These results are being reported for the first time.

\section{Experimental}

Highly pure zinc metal ingots obtained from the Solid State Physics Laboratory, New Dehli was used. The powder samples were obtained by gently filing highly pure $\mathrm{Zn}$ metal ingots with a jeweller's file. A part of this powder was used to prepare the initial sample. The remaining powder was subjected to milling in a high emerged ball mill for 3, 6, 9, 10 and 12 hours to produce strains and decrease particle size. Xray diffractograms were recorded with the initial sample and with samples prepared after each spell of milling. The diffractograms were obtained with a Philips CWU $3710 \mathrm{X}$ ray powder diffractometer in the $2 \theta$ range $20-120^{\circ}$ using filtered $\mathrm{CuK}_{\alpha}$ at a goniometer speed of $0.5^{\circ}$ per minute and a chart speed of $20 \mathrm{~mm} / \mathrm{min}$. All measurements were made at room temperature.

Figure1 The XRD paterns of $\mathrm{Zn}$ powders are given in Figure 1. The observed integrated intensities have been corrected for thermal diffuse scattering using the method of Chipman and Paskin [12]. 

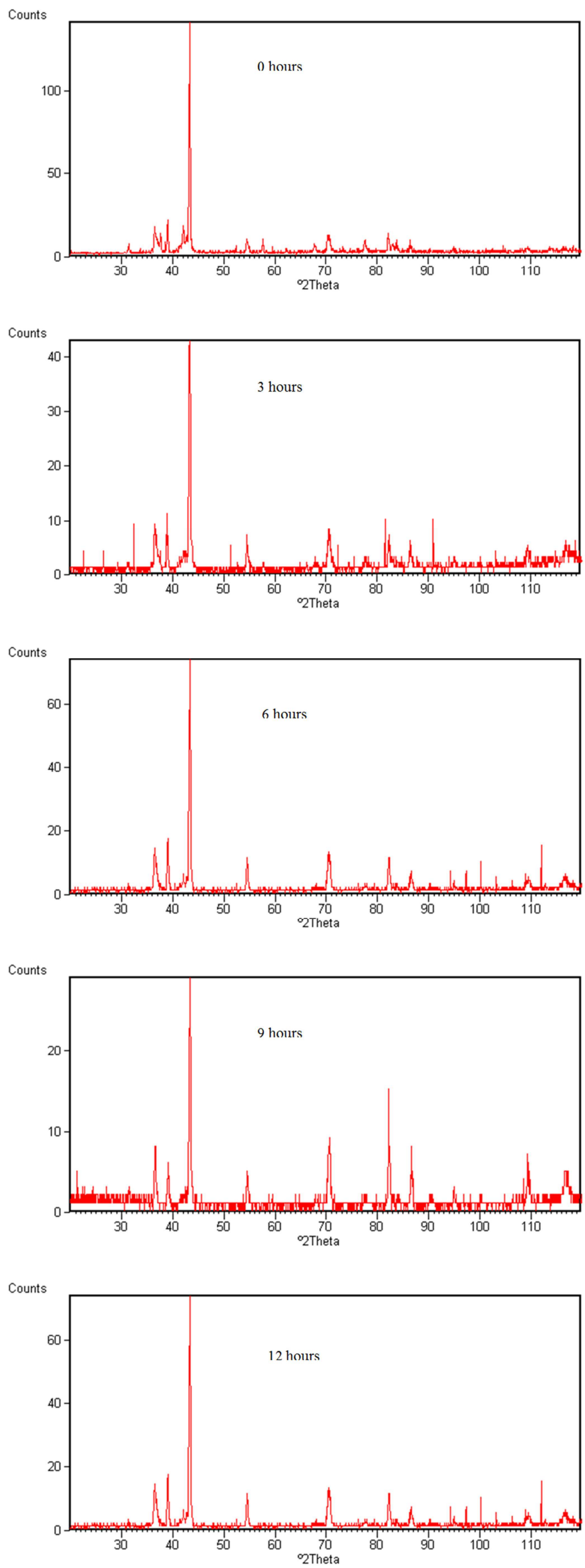

Figure 1. The XRD patterns of Zn powder. 


\section{Analysis of Data}

The procedure for the determination of directional meansquare amplitudes of vibration $\left\langle u_{\| 1}{ }^{2}\right\rangle$ and $\left\langle u_{\perp}{ }^{2}\right\rangle$ from the intensity data and the estimation of associated errors is as discussed by Gopi Krishna et al [13]. The average mean square amplitude $<\mathrm{u}_{\mathrm{av}}{ }^{2}>$ can be obtained from the relation,

$$
<\mathrm{u}_{\mathrm{av}}^{2}>=1 / 3\left(<\mathrm{u}_{\|}^{2}>+2<\mathrm{u}_{\perp}^{2}>\right)
$$

and the directional Debye-Waller factors $B_{\perp}$ and $B_{\|}$were obtained from the equations

$$
\left\{\begin{array}{c}
\mathrm{B} \perp=8 \pi 2<\mathrm{u} \perp 2> \\
\mathrm{B}=8 \pi 2<\mathrm{u} 2>
\end{array}\right.
$$

The mean Debye-Waller factor B is given by

$$
\mathrm{B}=\left(2 \mathrm{~B}_{\perp}+\mathrm{B}_{\mathrm{II}}\right) / 3
$$

The directional Debye temperatures $\theta_{\perp}, \theta_{\|}$and mean Debye temperature $\theta_{M}$ were obtained from $B_{\perp}, B_{\|}$and $B$, respectively using the Debye-Waller theory [14] relation,

$$
\mathrm{B}=\left(6 \mathrm{~h}^{2} / \mathrm{M} \mathrm{k} \mathrm{k}_{\mathrm{B}} \theta_{\mathrm{M}}\right) \mathrm{W}(\mathrm{X})
$$

$$
\left\{\begin{array}{l}
\mathrm{B} \perp=(6 \mathrm{~h} 2 / \mathrm{M} \mathrm{kB} \theta \perp) \mathrm{W}(\mathrm{X}) \\
\mathrm{B}=(6 \mathrm{~h} 2 / \mathrm{M} \mathrm{kB} \theta) \mathrm{W}(\mathrm{X})
\end{array}\right.
$$

Where $\mathrm{h}$ is the Planck's constant, $\mathrm{k}_{\mathrm{B}}$ the Boltzmann constant, $\mathrm{M}$ the atomic weight. The function $\mathrm{W}(\mathrm{X})$ is given by

$$
\mathrm{W}(\mathrm{X})=[\phi(\mathrm{X}) / \mathrm{X}+(1 / 4)]
$$

Where $X=\theta_{M} / T, T$ is the temperature of the crystal and $\phi(X)$ is the Debye function. The values of $W(X)$ for a wide range of $X$ can be obtained from standard tables [15].

Lattice strain and Particle size determination

When the size of the individual crystals is less than about $100 \mathrm{~nm}$ the term "particle size" is usually used. When the crystallites of a material are smaller than $100 \mathrm{~nm}$, they have too small a number of parallel diffraction planes and so they produce broadened diffraction peaks instead of a sharp peak. Lattice strain present in the sample is another cause of broadening of Bragg diffraction peaks. In addition to this, there are instrumental factors such as unresolved $\alpha_{1}$ and $\alpha_{2}$ peaks, imperfect focusing which lead to the line broadening. There are various methods in practice to estimate the particle size. X-ray diffraction is a simpler and easier approach for the determination of precise particle size and the lattice strain in powder samples. The principle involved in the X-ray diffraction approach is precise quantification of the broadening of the Bragg diffraction peaks. Scherrer equation, Hall-Williamson method and Warren-Averbach method are some of the techniques based on this principle. Of the above methods, Scherrer equation method for the estimation of particle size does not take into account the broadening due to lattice strain present in the sample. As such in the present investigation, the lattice strains have been estimated using Hall-Williamson method. Recently, Bharati et al [16] have used this method to estimate the lattice strain and particle sizes of silver nano particles and composite silver nano particles. In this method the integral breadth of the diffraction peak is determined. The integral breadth is given by the integrated intensity divided by the maximum intensity. Thus, the observed peak broadening $B_{0}$ may be represented as.

$$
\mathrm{B}_{\mathrm{o}}=\mathrm{B}_{\mathrm{i}}+\mathrm{B}_{\mathrm{r}}
$$

Where $B_{o}$ is the observed peak broadening in radians, $B_{i}$ is the instrumental broadening in radians and $B_{r}$ is the broadening due to the small particle size and lattice strain. The instrumental broadening has been estimated using a pure strain-free fine sodium chloride powder sample subjected to XRD under identical conditions as those for the strained metallic powders. Eq. (5) holds good if the diffraction peaks exhibit purely Cauchy profile. However, when the diffraction peaks are partly Cauchy and partly Gaussian for profiles, the following relation between $\mathrm{B}_{\mathrm{o}}, \mathrm{B}_{\mathrm{i}}$ and $\mathrm{B}_{\mathrm{r}}$ holds good, Bharati et al [16].

$$
\mathrm{B}_{\mathrm{r}}=\left[\left(\mathrm{B}_{\mathrm{o}}-\mathrm{B}_{\mathrm{i}}\right)\left(\mathrm{B}_{\mathrm{o}}{ }^{2}-\mathrm{B}_{\mathrm{i}}{ }^{2}\right)^{1 / 2}\right]^{1 / 2}
$$

Now, according to Scherrer equation, the broadening due to small particle size may be expressed as.

$$
\mathrm{B}_{\mathrm{c}}=\frac{\mathrm{k} \lambda}{\mathrm{t}} \cos \theta
$$

Where $\mathrm{B}_{\mathrm{c}}$ is the broadening solely due to small crystallite size, $\mathrm{K}$ a constant whose value depends on particle shape and usually taken as unity, $\mathrm{t}$ the crystallite size in nanometers, $\theta$ the Bragg angle and $\lambda$ is the wavelength of incident X-ray beam in nanometers. Similarly, according to Wilson [17], the broadening due to lattice strain may be expressed by the relation,

$$
\mathrm{B}_{\mathrm{s}}=\varepsilon \tan \theta
$$

Where $\mathrm{B}_{\mathrm{s}}$ is the peak broadening due to lattice strain and $\varepsilon$ the strain distribution within the material and $\theta$ is the Bragg angle. Based on Eqs. (7) and (8) the total peak broadening $B_{r}$ may be expressed as.

$$
\mathrm{B}_{\mathrm{r}}=\frac{\mathrm{k} \lambda}{\mathrm{tcos} \theta}+\varepsilon \tan \theta
$$

Which can be written as

$$
\mathrm{B}_{\mathrm{r}} \cos \theta=\frac{\mathrm{k} \lambda}{\mathrm{t}}+\varepsilon \sin \theta
$$

Figure 2 The plot of $B_{r} \cos \theta / \lambda$ versus $\sin \theta / \lambda$ is a straight line with slope equal to $\varepsilon$ and hence the particle size ' $t$ ' can be estimated from the intercept. Typical Hall-Williamson plot between $B_{r} \cos \theta / \lambda$ and $\sin \theta / \lambda$ is shown in Figure 2. 


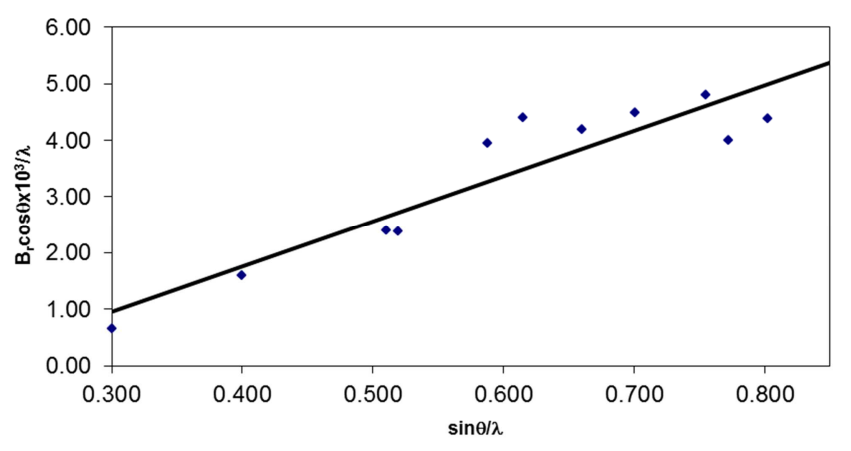

Figure 2. Plot of $B_{r} \cos \theta / \lambda$ Vs $\sin \theta / \lambda$ for Zn after milling for 9 hours.

The lattice strains were determined from the plot of $\mathrm{B}_{\mathrm{r}} \cos \theta / \lambda$ against $\sin \theta / \lambda$ following standard procedures [18]. The measured half-widths were corrected for instrumental broadening with reference to a pure strain-free silicon powder. The variation of particle size with milling time is within the limits of experimental errors. This shows that while the milling is enough to create strains, it affect the particle size to a measurable extent. A typical HallWilliamson plot is shown in Figure 2 for $\mathrm{Zn}$ after milling for 9 hours.

\section{Results and Discussion}

The values of the particle size, lattice strain, root mean square amplitude of vibrations, Debye-Waller factor and Debye temperature of $\mathrm{Zn}$ powders, ground for different durations, obtained in the present study are given in Table1. Although, values of Deby-Waller factor, amplitude of vibration and Debye temperature in the ' $a$ ' and ' $c$ ' directions have been determined separately, the average values of these quantities are given in Table1. As the objective of the present work is to investigate the strain dependence of Debye-Waller factors.

Table 1. Values of particle size ( $t)$, lattice strain $(\mathcal{E})$, mean Debye-Waller factor $(B)$, root mean square amplitudes of vibration $<u_{a v}>$, mean Debye temperature $\left(\theta_{M}\right)$ and energy of vacancy formation $\left(E_{f}\right)$ of strained nzno Zn powder.

\begin{tabular}{|c|c|c|c|c|c|c|c|}
\hline Metal & Milling time(hrs) & $\varepsilon \times 10^{3}$ & $t(n m)$ & $\mathbf{u}_{\mathrm{av}}(\AA)$ & $B_{\text {av }}\left(\AA^{2}\right)$ & $\theta_{M}(K)$ & $E_{\mathrm{f}}(\mathrm{eV})$ \\
\hline \multirow[t]{5}{*}{$\mathrm{Zn}$} & 0 & 0.64 & 210 & 0.014 & 1.11 & 219 & 0.47 \\
\hline & 3 & 1.54 & 118 & 0.016 & 1.40 & 201 & 0.38 \\
\hline & 6 & 2.47 & 72 & 0.020 & 1.62 & 184 & 0.32 \\
\hline & 9 & 2.95 & 46 & 0.021 & 1.80 & 178 & 0.29 \\
\hline & 12 & 3.38 & 34 & 0.022 & 1.86 & 171 & 0.26 \\
\hline
\end{tabular}

While comparing the Debye-Waller factors calculated from the lattice dynamical models with experimental results Vetelino et al [19] have attributed the difference to inaccuracies in the experimental values caused by neglecting the TDS corrections. The repeated milling of the powder sample leads to lattice distortion which gives rise to microstrains in the lattice. These microstrains increase the contribution of static component of Debye-Waller factor. Thus both lattice strain and the observed Debye-Waller factor, which is the sum of static and thermal components, increase with grinding time. Thus, whenever Debye-Waller factors are determined from X-ray intensities on powder samples, it is desirable, to make an estimate of the lattice strain and if the strain is large, a suitable correction is to be made as in the present study. The Debye temperatures derived from the zero strain values of Debye-Waller factor of $\mathrm{Zn}$ is $229 \mathrm{~K}$. This value agree well with the value of $222 \mathrm{~K}$ [13] obtained for $\mathrm{Zn}$ from the single crystal X-ray diffraction.

Glyde [20] derived the following relation between the energy of vacancy formation $\left(\mathrm{E}_{\mathrm{f}}\right)$ and the Debye temperature $(\theta)$ of a solid. The relation is

$$
E_{f}=A(k / h)^{2} M \theta^{2} a^{2 z}
$$

where a is the interatomic spacing, A a constant shown to be equal to $1.17 \times 10^{-2}$, M the molecular weight and $\mathrm{h}$ and $\mathrm{k}$ are the Plank's and the Boltzmann's constants, respectively. Glyde recommended the use of X-ray based values in eq. (11). The validity of eq. (11) was verified for a number of fcc, bcc and hcp metals [21]. Therefore, the X-ray Debye temperatures obtained in the present work have been used to study the variation of vacancy formation energy as a function of lattice strain in $\mathrm{Zn}$. The values of vacancy formation energies are also included in Table 1.

\section{Conclusion}

From a study of X-ray diffractograms recorded at different stages of milling, it is observed that milling for 12 hours has systematic effect on the particle size. However, the milling produces lattice strain and also enhances the effective DebyeWaller factor. By an extrapolation of the plot between the Debye-Waller factor and the lattice strain are obtained for $\mathrm{Zn}$. The variation of energy of vacancy formation as a function of lattice strain has been studied.

\section{References}

[1] Md. Imran Mohiuddin, A. Devaraju and B. Manichandra, International Journal of Materials Science, 12, issue.4 (2017) 599-605.

[2] M. Shiva Chander, P. Satish Kumar, A. Devaraju, International Journal of Mechanical Engineering and Technology, 8, issue.11 (2017) 327-334. N. Kavcar, Solar Energy Materials and Solar Cells 52 (1998) 183.

[3] P. Satish Kumar, Ch. S. R. Sastry, A. Devaraju, Materials Today Proceedings Elsevier, 4, issue.2 (2017) 330-335.

[4] V. Ashok Kumar, P. Sammaiah, Science Direct Materials Today, 1 (2017) 7.

[5] E. F.Skelton and J. L. Katz ,Phys. Rev. 171,801 (1968). 
[6] E. Rossmanith, Acta Cryst. A33, 593 (1977).

[7] M. Inagaki, H. Furuhashi, T. Ozeki et al., J Mater Sci. 6, 1520 (1971).

[8] M. Inagaki, H. Furuhashi, T. Ozeki \& S.Naka, J. Mater, Sci.8, 312 (1973).

[9] D. B.Sirdeshmukh, K. G.Subhadra, K. A.Hussain, N. Gopi Krishna, B. Raghave-ndra Rao, Cryst. Res. Technol, 28, 15 (1993).

[10] N. Gopi Krishna and D. B. Sirdeshmukh, Indian J Pure \& Appl Phys.31, 198 (1993).

[11] N. Gopi Krishna et al, Indian J Phys. 84 (7), 887 (2010).

[12] D. R. Chipman and A. Paskin, J. Appl. Phys. 30, 1938 (1959).

[13] N. Gopi Krishna, D. B. Sirdeshmukh, B. Rama Rao, B. J. Beandry and K. A. Jr. Gsch-neidner, Indian J Pure \& Appl Phys.24, 324 (1986).
[14] R. W. James, The optical principles of the diffraction of x-rays (Bell and Sons, London, 1967).

[15] International tables for X ray crystallography, Vol.III (Kynoch press, Birmingham) (1968).

[16] Bharati, R., Rehani, P. B., Joshi, Kirit N., Lad and Arun Pratap, Indian Journal of Pure and Applied Physics, 44, (2006) 157-161.

[17] Wilson, A. J. C., (1949). X-ray Optics (Methuen, London).

[18] Kaelble, E. F., Handbook of X-rays (New York Mc Graw ill) (1967).

[19] J. F. Vetelino, S. P. Gaur, S. S. Mitra, Phys. Rev. B5, 2360 (1972).

[20] H. R.Glyde, J. Phys and Chem Solids (G. B), 28, 2061 (1967).

[21] Micro-and Macro-Properties of Solids,Springer Series in Material Science, (2006). 